\title{
Empowering Leaders through the COVID -19 Crisis: Implementing a Transformative Communication Model
}

\author{
Claudine Bent-Cunningham ${ }^{*}$ and Nicole Mauzard, PhD \\ Northern Caribbean University, Jamaica \\ "Corresponding Mail: claudine.cunninghampml@yahoo.com
}

\begin{abstract}
This study was a reflection on the implementation of a transformative communication model at a school in Central Jamaica to address interruptions in communication and school operations among key stakeholders brought by the COVID-19 pandemic. The researchers sought to establish what kind of support the leaders and teacher leaders needed to improve communication among staff members and pupils in order to restore stability to the school environment. The project employed the Kurt Lewin's change model: Unfreeze, Change, re-freeze. The study involved seven participants: the leader of the institution and six teachers who operated as teacher leaders with varying leadership portfolios. The researchers found that crisis leadership was necessary to mitigate against the impact of the COVID-19 pandemic on the institution which existed in the deep rural community. The researchers also found that improving leader' and teacher leaders' communication competencies boosted morale and encouraged normalcy in school operations. In applying the transformative communication model, the institution was entirely transferred to the TEAMS platform. A team of international experts in education and communication, that became a sustained extension of the institution, trained the leader and teacher leaders to manage the crisis and increased their communication competencies, while normal school operations continued on the platform. This project demonstrated that in times of crisis, if all stakeholders collaborate, organizational goals can be attained.
\end{abstract}

Keywords: Crisis Communication, Crisis Leadership, Communication Model, COVID-19 pandemic, Transformational Leadership

"I've learned that people will forget what you said, people will forget what you did, but people will never forget how you made them feel" Maya Angelou.

\section{Introduction}

From times immemorial, communication has had a primary importance in the affairs of mankind. Since it is through effective communication that we construct meaning from our numerous interactions with each other and with the environment, communication may be regarded as the heart of leadership. Motschnig and Ryback (2016) emphasized this idea as they suggest that communication and leadership are the most vital skills in today's workplace and are intricately intertwined. The authors posit that a leader or manager cannot be effective without having the requisite communication skills as well as clear articulation and good listening skills. Modzelewski (2019) reiterates this view when he states that there are both rewards and risks in leadership communication. He purports that leaders use communication to influence urgency, energy and inspiration.

Modzelewski further states that leadership communication serves the purpose of clarifying mission, giving direction and ascertaining purpose which will promote collaborative partnerships. He posits that communication gives leaders the springboard to achieve their goals. Effective communication, he believes, is forging partnerships, influencing followers and appealing to their intelligences, as well as their hard and soft skills to drive the organization forward. 
By exploring the work of a plethora of leaders in the field of communication and leadership, a Transformative Communication Model with a threepronged approach was adopted to solve issues with communication in one primary school amid the Covid- 19 pandemic. The three arms of this model include: Innovation and Communication, Emotional Intelligences and Transformational Leadership. The work of Carl Rogers (1979) was also utilized in this project. Known for his Person-Centered Approach (PCA), Rogers has made central, individuals' feelings and emotions, to arrive at a place of trust between the leader and constituents. Rogers has therefore shifted the paradigm in organizational communication from the traditional hierarchical to the more contemporary collaborative approach. It is in this regard that a collaborative approach, through networking between leaders and followers, was interwoven into this project.

In implementing the transformative communication model, the researcher identified an institution within the Jamaican setting which demonstrated a need for communicative change and implemented the model through the Kurt Lewin's Change Model. The following is a reflection on the implementation process and an evaluation of the program in meeting the needs of leaders and teacher leaders at the specified institution. According to the researchers' knowledge, a study of this nature has never been done in the Jamaican setting. Research conducted in this area can add to our overall understanding of the impact of the COVID-19 pandemic on one rural primary school, and it gives a better picture of how leaders, teacher leaders and students were affected by the crisis.

On hearing the testimonials from many leaders and teacher leaders about the difficulties with communication brought about by the COVID-19 pandemic, in particular, from an educator who leads a school in deep rural Jamaica, the researchers were moved with compassion and decided to research and implement a transformative communication model which could help the leader and future educators to better handle the crisis of the covid-19 pandemic and also to maintain a crisis leadership culture within that school. Educational leaders had the daunting task of switching to the crisis leadership mode while dealing with the effects of the pandemic. Collaboratively, leaders and their teams were faced with the tasks of moving the teaching and learning environment to the electronic platform amidst the lack of technological tools and infrastructure as well as preparing teachers and staff for the online interaction which required hard and soft skills which were initially lacking for teachers and students (Fernandez and Shaw, 2020).

The Director General of the World Health Organization (WHO), Ghebreyesus (2020) in a statement urged nations to collaborate internally and externally against the pandemic which was foreshadowed to cause devastating effects worldwide. He further asserted that the Covid-19 pandemic has drastically altered, possibly indefinitely, human life, public health, food systems and has tested humanities resilience to its very core. This is indeed evident in the ways we now deal with schooling, working, worshipping and shopping. We have felt the demoralizing results of the world faced with yet another deadly pestilence which has hit hard at the core of our existence. Leadership at all levels has been negatively affected primarily through increased virtual communication and a strange kind of 'absence'. Very suddenly, Social Media exited the realm of entertainment and leisure to become vital to our survival. Consequently, all institutions including schools and homes had to get on board or be quickly decimated. This project was guided by one research question namely; what factors hindered leaders and teacher leaders from operating effectively during the COVID19 pandemic crisis?

\section{Review of Literature}

In this section, the researchers reviewed what writers opined about the topic of crisis management and crisis communication to give credence to the response taken to implement the transformative communication model to solve the crisis that the school leader and teacher leaders experienced during the Corona Virus (COVID-19) pandemic.

According to Zaremba (2010), the skillsets needed for the crisis communication leader include the ability to speak under pressure and respond effectively to questions, write effectively, conduct meetings with the communication crisis team and stakeholders, utilize new and innovative technologies to enhance communication and receive feedback. Another important feature that is evoked in the crisis communication literature is the linking of the organization through networks. Constituents must maintain links with their leaders and leaders should be able to take quick decisions on behalf of constituents and the institution. Since in a crisis individuals may not be able to move about 
freely, it is crucial that the organization develops a network system where communication remains smooth even while constituents are at different locations. This network should be in place long before the crisis occurs.

Ndlela (2019) posits that crisis management has moved from a narrow perspective to a broader one. He purports that crisis management no longer looks at merely solving the crisis, but it seeks to deal with the crisis in three phases. Mackey (2015) concurs that these phases include the pre-crisis phase, crisis response and the post -crisis phase. In reviewing the work of Jacques (2007), Ndlela established another cycle which entails a four-pronged approach. In this model, Ndlela discusses the steps to include crisis preparedness, crisis prevention, crisis incident management and post-crisis management. The first two, the author believes, can be taken together; that is, preparation and prevention; while the last can be taken together with the first, that is, postcrisis management and crisis preparedness. Altogether, he sums, each element is critical to the crisis management cycle. The author further adds to the conversation, the potency of crisis communication to the crisis management process by suggesting a parallel between both cycles. Like crisis management, crisis communication does not exist during the responsive wrung of the cycle but is entrenched throughout the entire duration of the crisis, from preparedness to post-crisis. The approach has been broadened by other fields including psychology, communication and media and organizational studies.

Mehta and Greer (2015) employ the term risk communication to offer their views about the role of this type of communication and how it is encapsulated in the crises phases. In sum, they define risk communication as 'information disseminated to individuals or groups to instruct, develop awareness, inform decisions and alter attitudes and behavior during the crises or disaster' (pp.160-161). Disaster management agencies in Australia, according to the writers, usually divide their disaster plans into four tranches: prevention, preparedness, response and recovery, and risk communication which are pivotal across all spectrums for effective crisis management to occur.

Mehta and Greer (2015) in reviewing the work of Sturges (1994), extrapolate on the three phases of communication that are inherent in risk communication during a disaster. The first stage is referred to as the instructional stage where individuals are instructed how to handle the crisis. This stage involves certain responses in attitude and behavior. The second phase is the adjusting phase which aids individuals in psychologically dealing with the crisis. The third phase is the internalizing phase which helps constituents to remain positive about the crisis. The first stage is crucial across all four disaster phases despite it being most critical during the preparedness, response and recovery stage. The work of Sturges (1994) is relevant in grounding the importance of risk communication in steering the organization through a crisis.

Advani (2020) cited in Goldsmith and Osman (2020) writes his views on ways managers and stakeholders in organizations should react to the crises of the COVID -19 pandemic. His views also included schools and school leaders. The author begins by stating that CEOs and managers should immediately address challenges or turn challenges into opportunities during the crisis. He stated that 100 schools globally, supported by his organization experienced school closure in 130 countries bringing their work to a slowed pace. He further states that his organization and leaders have had to convert quickly to the electronic space in order to keep the business of education functioning.

In maintaining the communication flow among companies served by Advani's organization, board members divided themselves into teams at the local, national, regional and global levels in order to serve in these areas more effectively. This organizational structural division he believed aided with the smooth running of the company amidst the pandemic. Leadership roles became modified to respond to urgent questions such as finance, innovation and marketing which needed immediate decisions and consensus. The author outlines a list of steps for crisis leaders, adopted from a document prepared by a United States law firm, Watchell Lipton, and IESE Business School in Spain, and which was employed by his own organization.

The document proposes that leadership must have a crisis management plan. Budgets must also be revised to include shifts in operations and considerations made for the health of constituents as well as the health of the company. Leaders should also implement and participate in communication plans both internally and externally. They should also initiate opportunities arising from the crisis and adjust the organization to their new realities and maintain positive relationships among members. These ideas postulated among the 
authors on crisis and risk communication will be adopted in the implementation of the transformative communication model.

\section{Methodology}

The research was an implementation study which sought to ascertain the deficiencies experienced by leaders and teacher leaders in carrying out their expected duties during the COVID-19 pandemic. The implementation research is a method which aids educators in accomplishing their goals of creating lasting changes by propelling theory into practice through interventions and innovations (Century and Cassata, 2016). The SWOT analysis and testimonials from participants were employed to fully determine the need of leaders and teacher leaders within the institution. The study used a single group of leaders and teacher leaders from one primary school in a rural community in Central Jamaica to understand the response that was needed to address deficiencies that the educators experienced. The intervention provided teachers with a series of professional development training towards implementing the transformative communication model as well as the launch of an electronic platform from which the school will operate fully in times of school closures. The process of implementation will occur through the Kurt Lewin's (1951) Change model. A survey was used to evaluate the process after implementation.

\section{Demographic Information}

School $X$ is a government owned institution located in deep rural Jamaica. It is a second-tier institution built to accommodate a population of 370 pupils. During the time of data collection, the school had 9 teachers and one principal. Included in the cadre of teachers are two guidance counsellors. Two teachers were trained senior staff members and two were junior teachers training for seniority postings. Other teachers held positions of varying minor leadership responsibilities. The school had seven classrooms and offices. Additionally, there was a poultry house, kitchen, library and computer room. The institution relies on tanked water through rainfall or purchased water to fulfill daily water needs. The school existed in a farming community, and the school and the community had a symbiotic relationship. The school depended on the community for resources and protection while the community depended on the school for training of adults who are offered secondary and tertiary programs electronically through local community colleges.

\section{SWOT Analysis}

A mammoth part of crisis management intervention was to first determine the issue which faced the institution. This was best done through the SWOT analysis (Diagram 1) which is about the strengths, weaknesses, opportunities and threats that the institution faced. The researchers thought it best to display from the SWOT analysis the weaknesses and opportunities of the institution only, so that the readers might follow the transformation process that will be implemented in response to the crisis facing the school.

\section{Diagram 1: SWOT analysis Results}

\section{Weaknesses}

- Inconsistent Internet use and connectivity resulting in constant disconnect in communication among stakeholders

- School exists in a remote location which makes reach difficult

- Poor regional support for remote learning

- Un-sustained training and practice

- Inadequate resources
- Training in Google suite

- Professional upgrade and training in technology to support remote teaching and online delivery

- Exposure to and use of different teaching modalities

- Peer support and study group meetings to influence groupings

- transformational leadership and crisis management by principal

- $\quad$ internal and external workshops on communication and emotional intelligences

- Mentoring workshop and coaching
One major weakness the school faced was in regard to internet usage and internet connectivity which resulted in constant disconnection in communication among stakeholders. This disconnection in communication affected the school's operations because the school existed in a 
remote community which was sparsely populated; hence, disruptions in communication severed stakeholders from accessing the schools' services. For some teachers, when the school was locked down due to the risk of COVID -19, they lost connection with students, colleague and parents. Two teachers were unable to participate in this research as a result of the issue. A few other teachers had issue with internet use. From the testimonials in the what's app group, one teacher expressed that she had problems with screen sharing, creating google forms for tests and surveys as well as creating breakout rooms within the google classroom.

Another teacher stated that she had difficulties taking register in google classroom and inserting videos and audios. A third teacher said she was not savvy with the technology at all and welcomed any information that was available. The ideas expressed by the three teachers sum the volume of issues with technology that the entire group grappled with. Another major weakness that was cited was the issue of teacher training in technological tools. From the SWOT, the researchers learnt that at the time of this research, teachers did not have adequate training in the use of technological tools; as such, their inadequacies in managing the technology to bridge the gap in communication with students and colleagues contributed to disruptions in daily operations of the school..

The researchers utilized the information in the column entitled Opportunities to implement the transformative communication model. In this column the institution felt that teachers needed training in google suite and other technologically advanced tools to develop communication skills for remote teaching, peer group meetings and school meetings. In addition, to the communication skills outlined, the researchers also garnered from the SWOT that soft communication skills were needed to improve the way leaders and teacher leaders communicated with each other and with the macro community during the crisis.

\section{Transformative Communication Model}

The transformative communication is the proposed model by the researcher and a team of experts that was implemented at School $X$ to strengthen weaknesses and turn them into opportunities as it relates to communication. From the list in the SWOT provided by the school, the researcher and the team attempted to strengthen communication competencies and social relations to bridge some key communicative issues which can occur during a crisis.

At the center of the communication model is Carl Rogers' Person Centered Approach (PCA). The idea for this approach came out of Rogers' experience with individuals and clients at his organization. He postulated that while the practice has found its genesis in his counselling sessions, it can be applied to relationships on the whole where there is the constituent and an individual who holds a valuable position of trust (Rogers, 1979). According to Rogers, the ideology of the person centered approach operates on the premise that individuals have the ability to understand themselves, alter attitudes and self- direct their behaviors if the appropriate climate is provided. He propels three conditions which contribute to this growth. Firstly, there should be a climate of congruence and genuineness between leadership and constituents; secondly, there should be acceptance in the relationship between both parties. Rogers purports that these factors prepare the conditions for change, and that there should be the empathetic understanding where the leader is sensitive and attune to the feelings of the constituents. Motschnig and Ryback (2016) refers to this kind of empathy as active listening, an idea he believes was adapted by contemporaries like Goleman (1995) who used the ideology as one of the components in his model on emotional intelligence.

\section{Emotional Intelligence}

Furnham (2012) opines that the literature on emotional intelligence is emerging. In reflecting on works conducted within the field, he explains that the idea of emotional intelligence existed as far back as the 1920's but was popularized by Goleman (1995) in his publication: 'Emotional Intelligence.' Panait and Bucinschi (2018) attribute the theory of emotional intelligence in its most early form to the writings of John Mayer and Peter Salovey who sought to conduct research on individuals from an ability perspective. The two professors discovered that individuals were able to express their own feelings with those around them and also solve problems relating to their emotions. Since then, the field of emotional intelligence has become so vast that "a google search in the field could result in up to 7 million hits" (Furnham, 2012, p.22). In defining emotional intelligence, Goleman in an interview with O'Neil (2002) states that emotional intelligence is a different form of being smart. It involves

161 East African Journal of Education and Social Sciences (EAJESS) 2(2)157-168. 
identifying with one's feelings and using these to make decisions about one's life. Additionally, he states that it involves handling stresses and irrational thoughts to realize positive outcomes. Finally, he includes in his definition the need for empathy that understands the feelings of others having positive relationships and being able to persuade and enjoy the trust of others.

Rathore, Chadha and Rana (2017) purport that having the skills of emotional intelligence among individuals in the workplace can indeed transform the work environment. The authors state that this can build both 'valence' and 'focus;' the former having to do with positive and negative emotions and how these are expressed and understood and the latter having to do with tasks whether emotionally, socially or task related. According to Dubrin (2016), Daniel Goleman, based on research done in multiple studies found that effective leaders had a high degree of emotional intelligence. As such, many leaders with a high degree of emotional intelligence become resonant leaders.

In applying emotional intelligence to the field of leadership, Dubrin (2016) outlines some key characteristics of the resonant leader. The first of which is self- awareness whereby leaders are effective when they understand their own emotions and are able to understand how their actions affect others. Secondly, self-management, the ability to control one's emotions and act with honesty and integrity. Thirdly, social awareness which is one of the key traits that include empathy for others and intuition to solve organizational issues. Finally, of utmost importance is relationship management. This is where the leader is required to have strong inter- personal skills, communicate effectively, clearly and convincingly, lessen conflicts and forge strong partnerships. It is these traits, developed in various tranches or combined, which result in behaviors that drive the success of any given organization, especially during a crisis.

\section{Communication and Innovations}

Communication whether technological, verbal or non-verbal plays a critical role in organizational leadership as leaders lead through their words and actions (Barrett, 2014). Barrett states that an effective leader demonstrates good communication skills which include the ability to enable, foster and create positive relationships resulting in constituents' trust. An effective leader must have a clear vision and mission (Stacks and Wright, 2014) and must articulate these in varying and consistent ways within the organization. The leader must inculcate shared values within the organizational system (Kouzes and Posner, 2017), which is usually attainable by forging healthy partnerships, affirming, uncovering, reinforcing and allowing constituents to hold each other accountable based on what the organization values.

Communication necessitates interaction between individuals and groups (Unsar, 2014). As such, the medium is not employed by the leader only. Its use is also important among members and stakeholders. Effective communication aids in driving organizational goals among all stakeholders through varying channels; with members at some point exchanging leadership roles, depending on the thrust of the organization at that particular period. Forms of communication may vary according to the context in which the organization exists. Littlefield and Sellnow (2015) introduces these varying types of communication in what he calls relational dialectal theory (RDT), which is the study of how meanings are understood between or among individuals in relationships. It subscribes to antithetical emotions such as "connectedness and separateness, openness and closeness, certainty and uncertainty" (p.1). Varying perspectives are brought to the fore and members have the task of communicating differences of opinions while remaining positive and maintaining positive relationships during the respective work climate. The RDT is an ideal approach for relationship building, since in the face of a crisis, communication is required to be quick and efficient among stakeholders. Subsequently, the RDT can only be effective within organizations if communication soft skills are developed among members to ensure the smooth flow of information, interaction and interpersonal relationships within channels (Littlefield \& Sellnow, 2015).

Technology has now formed an integral role for effective communication in organizations. Before the COVID-19 pandemic, technology enabled learning; now the education sector depends on it (Diaz \& Lee, 2020). Adapt or die are daunting words that became a prominent reality for the $21^{\text {st }}$ century educators who had to adjust and re- adjust to synchronous and asynchronous learning heightened by the COVID-19 pandemic. Educators and pupils were plunged into the technological realm with little or no expertise to align both their soft and hard skills quickly and sufficiently to what some

162 East African Journal of Education and Social Sciences (EAJESS) 2(2)157-168. 
researchers are calling a fourth wave digital revolution (Rebrina, Khakimova and Ishkinyaeva, 2019). Leaders and teacher leaders have had to resort to innovative solutions in google suite such as google teams, google meets, google classroom, gdrive and Gmail to quickly solve the issue of communication during this novel crisis situation (Dhawan, 2020).

\section{Transformation}

Transformational leaders are value driven, purposeful and are viewed as heroes with charismatic personalities with a mission to drive the organizational change (Basham, 2012). The cry for innovation and organizational change has pealed across classrooms, staffrooms and boardrooms especially since the arrival of the COVID-19 pandemic. Anderson (2017) suggests that educational leaders, in order to facilitate the urgent call for change, need to benefit from the training which models transformational leadership styles used in business organizations.

Anderson further states that transformational leadership is ideal for establishing new norms, changing constituents' attitudes and developing positive cultures to combat crisis within the organization. Warwick (2011) supports this paradigm by looking at the transformational leader's ability to see through more innovative and entrepreneurial lenses which will enable them to remain competitive in an increasingly globalized society. The scholarship on transformational leadership can be utilized to drive the change in School $X$ that is needed to transform communication.

\section{Lewin's Change Model}

Change management sets out to alter the behavior of individuals in an organization or institution for the survival of that organization or institution (Lock, 2019). In seeking to propel School X's continuity, the study sought to transform the weaknesses of the institution's communicative system into opportunities by utilizing Kurt Lewin's (1951) Change Model. Lewin's Change Model has three phases: unfreeze, change and refreeze.

In the unfreeze stage, individuals or constituents recognize that there is a need for change, while in the change stage, the group searches for a novel way of approaching issues. Finally, in the refreeze stage, the new strategy is implemented and continually evaluated until it becomes part of the culture of the organization (Yukl, 2010).

\section{Unfreeze}

Through knowledge of the demographical information of School $X$, the study sought to establish the macro community as a context within which the educational institution existed. The researcher conducted a SWOT analysis to determine the needs of the institution. Hall (1976) purports that an organizational culture can be likened to an iceberg. Some aspects of the culture lie above the surface but beneath the surface there is a colossally concealed portion of values and thought patterns; hence, it is important to take the time to understand the individuals from that culture before certain assumptions are made.

Once a clear understanding of the school's challenges was determined, the researchers' first step involved a holistic approach (Worley and Vick, 2005) which involved engagement with all stakeholders of the institution. A debriefing letter was sent to the principal of the institution. This resulted in several engaging conversations of the planned project to solve some of the major issues the institution was having with communication among stakeholders due to the sudden interruption of regular schooling caused by the COVID-19 pandemic. The researchers then sent a proposal to the principal with the complete layout of the project and a timeline. Having perused the document, the principal and the researchers had further engagements about the process. The principal agreed that the project was exactly what School $X$ needed in order to stabilize the institution during a very turbulent period.

The principal began sensitizing the school's staff and other stakeholders about the project by discussing the problems the school was having and by proposing how the project would help in solving their own personal issues as well. The project was also discussed at the school board level, after which it was voted and accepted by the school's Board of Governors. The first direct communication with the staff was in the form of an effective communication checklist where all staff members were open about their current communication competencies. The other mode of communication was through a WhatsApp group where teachers texted exactly what they believed the problems were from their perspectives. This interaction with the staff gave the researchers a very clear idea of how they would ensure appropriate solutions to the communication issue were rendered. 
As part of the innovation of amalgamating strategies from the business literature and the field of education, the researchers began working on forming a strategic and innovation team (Dubrin, 2016) that would become an extended arm of the school body with an intended sustained life. In the selection of participants for the strategic and innovative team, individuals were chosen from relevant experts' fields worldwide. The researchers engaged each person individually and shared their vision for the project. They also shared the proposal with each prospective team member and listened for their feedback (Kouzes and Posner, 2017). All the team members adopted the project as their own and they were all very elated to join a team that would help to solve the problems currently faced within this educational institution in rural Jamaica. The team consisted of five persons, four of whom had expert skills in digital communications.

The researchers also had other persons who joined the team and who would add their expertise when this was needed. To facilitate group dynamics, they had several team meetings. It was fascinating to watch the interaction among them. All team members were sent the proposal before meeting. The proposal also consisted of the biography and picture of each team member. Hence, members communicated with much familiarity.

A formal invitation was sent to the principal to meet with the team on the zoom platform. The principal was most elated to meet the team that would be helping the school rise above its current challenges. On the day of the meeting, the team met on the zoom platform for two hours. In this meeting, the researchers served as lead moderators with the principal. They spoke about themselves and about the project, and the principal spoke about her school. The team had several meetings of this kind and as they communicated through the principal to the staff, the group developed a keen sense of awareness of the school's and teachers' needs. Each group member was assigned their specific task in preparation for a two-week training and implementation of the Transformative Communication Model.

The team began by advertising the project. Electronic flyers were created and sent to each stakeholder inviting them to join the training which was being organized by the principal and our team. The principal continued to sensitize and interact with the team of leaders and teacher leaders about the change which was about to take place. All stakeholders were contacted for their business information, which included: their email addresses, position held and any additional information about their leadership roles within their institution. The team then used this information to transfer the entire institution to an online platform. This move was facilitated by the principal and at the end of the transfer, the school then had a digital space where it continued its operations while marooned in their homes from the terror of the COVID-19 pandemic. Hence, while the school gates were closed and the school yard vacated, the school was in full operation in a virtual home. Stakeholders were placed in various channels where the groups continued to meet for classes and for group meetings. Conversations were direct and communication was immediate. There was also a channel for the team where they could be accessed by the principal and the staff according to their needs and according to the expert aid that the team could offer as a means of continuity (Venus, Stam \& Knippenberg, 2018). The team then began preparing for the training in areas postulated by the Transformative Communication Model.

\section{Change}

Teachers were first trained to use google meets and google teams to improve channels of communication within the organization. These trainings occurred in two sessions where the trainers joined the school in both instances on the identified platforms. The team presented to the entire staff and walked them through the process of how each platform could serve them holistically. The sessions were very interactive, teachers were comfortable with the team and this collaboration resulted in teachers understanding the technology more. They were now more positive about using it because they had understood many of the features which at first seemed very difficult to them. They got opportunities to practice together with the features, make mistakes and learn from these mistakes together (Sawyer, 2008). The teachers also stated in their testimonials that they felt more equipped to use the technological features to enhance their lessons and daily communication and their interaction with their colleagues. The team uploaded all the information and training material in the Teams portal that stakeholders could return to for reflective practice and reinforcement.

In the second week, the team invited an expert guest presenter from a local university to conduct training sessions on Transformational Leadership 
and Emotional Intelligence. These two concepts are key elements in developing effective leaders and those they lead in a futuristic way. The team was present along with the staff for these two sessions. The first session on transformational leadership was key to stakeholders since they were currently experiencing the negative effects of the COVID-19 pandemic, which deeply affected usual operations at the institution; the staff saw the need to be transformed.

Individuals responded positively to this training. Approximately $95 \%$ of the teachers demonstrated in their testimonials that they felt empowered as leaders and teacher leaders. They also registered their appreciation on the explication of the 4 I's (idealized influence, inspirational motivation, Intellectual stimulation, and Individual consideration) of the transformational leadership and suggested that they accepted these characteristics as traits needed to be acquired to lead the well needed change in their own institution (Northouse, 2001). The teachers got the opportunity to talk about how they felt about their current situation (Couch, 2020) and also got the opportunity to ask questions about how they could contribute to the change needed to drive their institution forward. Most teachers were positive about the opportunity to change and succeed while a few simply remained quiet. One teacher commented that all the traits discussed in the transformational leadership was evident in their principal whom they believed was working assiduously to guide them through the crisis. They posited that the leader was a strong role model who empowered the constituents, was a visionary and had made a myriad of changes in the institution and community (Farnsworth, et al. 2019).

On the final day of the training, the team did two presentations on Emotional Intelligence and the Importance of Communication Networks. These two sessions occurred on the same day so that stakeholders could benefit from understanding their emotions and appreciate the feelings and opinions of others, as well as understand how communication forms a vital component in affecting the feelings of those they interfaced with. The teachers found the session on emotional intelligence to be quite exciting and innovative. They spoke some more about their own feelings and highlighted the importance of acknowledging the feelings of others. In the talk back session, the teachers admitted that they would be more careful on how they related to each other. They also maintained that they would become more in touch with their own feelings, and also take into consideration the feelings of their colleagues.

The presentation on Communication Networks also strengthened the conversation on emotional intelligence, since emotions most times communicate what people want to say long before the spoken word is uttered. The team discussed with teachers the importance of verbal and nonverbal communications in the workplace. The discussion helped educators to understand that individuals are affected not only by what is openly stated but also what is communicated through gestures (Sinha \& Sinha, 2007). Individuals were encouraged to ensure that positive messages are sent both verbally and through writing, whether manually or electronically. While the information was not entirely new for the staff, it helped in stimulating and creating awareness among staff members. Some members stated that they would be more intentional about how they communicated with their colleagues, with students and with parents. Other teachers disclosed that they would practice what they had learnt in communication through networks to build rapport and contribute to the smooth running of their institution.

\section{Refreeze}

The Transformative Communication Model was fully implemented in School $X$. During the period of implementation, the school was inspected by the National Educational Inspectorate (NEI) which gave the project a positive review for its creativity and innovativeness. Communication was much more effective and fluent. The empowered leader and teacher leaders continued to schedule meetings, lead events, plan for their students and improve their skills. Teachers were less concerned about the debilitating effects of the pandemic on the institution because they had found a way to overcome some of their major obstacles.

The team ensured that all materials needed for reinforcement was placed in the resource channel on the Teams portal. Some teachers had apprehensions about things going back to how they were before the COVID-19 pandemic begun. The concerns were expected, as change is sometimes slow, but it is most times inevitable (Couch, 2020). As such, the empowered leaders latched on to the change because it was working for them. All participants received their certificates of achievement at the end of the training. The team

165 East African Journal of Education and Social Sciences (EAJESS) 2(2)157-168. 
also remained accessible to all stakeholders through the channel created for technical support. Training for teachers and other stakeholders would be further conducted by the principal and middle managers who received additional training on the Transformative Communication Model throughout the project. The team continued to work with the school to ensure that the institution's needs are being met as this culture of transformative communication is nurtured.

\section{Evaluation}

Approximately $95 \%$ of participants responded positively to the model. More than $90 \%$ of the leaders and teacher leaders responded that they were now much more comfortable with computer programs such as google teams and google classroom. Regarding the use of the electronic space, $85 \%$ of leaders and teacher leaders agreed strongly that their confidence had grown while less than $20 \%$ remained in between. All participants agreed that they saw the need to be more careful when communicating with others. The leader and teacher leaders felt for the most part that they were in touch with their own emotions having experienced the training, while $20 \%$ stated that they needed more practice in understanding how their own emotions affected others.

The majority of educators responded that they did not find the process of change to be very difficult, after the training in areas which the model promulgated was conducted. They were very appreciative of the project as it helped in solving the grave issues they had, adjusting at first to the changes that the pandemic abruptly brought. Less than $20 \%$ of respondents however, expressed that they were still trying to accept the change. More than half of the educators agreed that they were very dedicated to the team and this is usually reflected in their work ethics and how they relate to each other. The researchers were of the view that majority of the leaders and teacher leaders would work to sustain the model.

The empowered leaders and teacher leaders were proud to identify with the institution; this was a crucial tenet for the change. When individuals do not wish to identify with the institution, they can become saboteurs, and this would make change very difficult. They also stated altogether that their dress, speech, writings and gestures reflected their zeal for the success and transformation of their school. Despite the zeal of the majority, there were still approximately $5 \%$ of teachers that were not as accepting of the changes as the others. These indicated that they would prefer to go back to how they were before the pandemic.

The principal and the team planned to continue working to create an inclusive and positive environment for those leaders and teacher leaders who were still mourning the loss of normalcy. The final response encapsulates the relevance of the implementation of the Transformative Communication Model at School X. All participants resoundingly agreed that the project was very relevant to them, including those persons who were still grappling with the change. In this regard, it can be posited that the project had begun to generate some of the desired results and will serve to propel further growth and development in School X once the change is appropriately monitored and sustained.

\section{Conclusions}

The Transformative Communication Model has been proven to be very effective in creating more awareness and positive practice to stem some of the issues that School $X$ grappled with in response to the adverse effects the institution faced with communication which was brought on by the COVID- 19 pandemic. Teachers' knowledge of technology was increased, and they were better able to meet the demands of their communication needs in the face of a crisis. Additionally, teachers were more in tuned with their emotions and they stated that they were more aware and empathetic towards those with whom they worked. Greater awareness of individual feelings has proven to foster greater collaboration and a more harmonious environment. With the principal and the team at the helm leading and working with the empowered leaders and teacher leaders, transformation became evident.

Teachers acknowledged the need for the change that they were experiencing and they all concurred that their principal's style of leadership was fundamental to the acceptance of this change. The staff admitted that the principal was visionary, inclusive, participative and innovative which encouraged their own work ethics and commitment to the institution. Altogether, the model slowly became part of the culture of the institution and undoubtedly it may become entrenched with the appropriate monitoring and continuous evaluation. The researchers recommend that educators receive 
continuous training in technological tools to maintain relevance in an increasingly changing digital platform. Policy makers should set criteria for the development of a crisis manual in educational facilities for effective crisis management. Principals and teacher leaders should receive ongoing training in Crisis Communication.

\section{Acknowledgment}

The researchers would like to acknowledge the invaluable contribution of the following persons to this work: Ms. Marcia Bent, Ms. Sabrina St. Rose, Mr. Omar Anderson, Mrs. Kimarley Walker-Medley \& Dr. Marcia Thomas- Powell.

\section{Reference}

Anderson, M. (2017). Transformational leadership in education: A review of existing literature. International Social Science Review 93(1),113.

Barrett, D. J. (2014). Leadership communication. $4^{\text {th }}$ Ed, Mc Graw Hill.

Basham, L.M. (2012). Transformational leadership characteristics necessary for today's leaders in higher education. Journal of International Education Research 8(4), 343-348.

Century, J. \& Cassata, A. (2016). Implementation research: Finding common ground on what, how, why, where, and who. Review of research in education 40(1), 168-215.

Couch, N. (2020). Communication and transformational leadership during a crisis. Communication and transformational leadership during a crisis - American Nurse. From myamericannurse.com.

Dhawan, S. (2020). Online learning: A panacea in the time of covid-19 crisis. Journal of Educational technology Systems 49(1), 5-22.

Diaz, M. M., \& Lee, C. (2020). A silent revolution in What technology can and can't do for Education: A comparison of 5 stories of success. Barbara Karni, Ed. Inter- American Development Bank.

Dubrin, A., J. (2016). Leadership: Research findings, practice, and skills, $8^{\text {th }}$ edition, Cengage Learning.

Farnsworth, D., Clark, J. L., Hall, J., Johnson, S., Wysocki, A., \& Kepner, K. (2019). Transformational leadership: The transformation of managers and associates. https://edis.ifas.ufl.edu.

Fernandez, A. A., \& Shaw, G.P. (2020). Academic leadership in a time of crisis: The coronavirus and covid-19. Journal of Leadership Studies 14(9), 39-45

Furnham, A. (2012). Emotional Intelligence: New perspectives and applications. Research Department of Clinical and Health Psychology.

Ghebreyesus, T.A. (2020). No end in sight to COVID crisis, and its impact will last for 'decades to come. https://news.un.org/en /story/ 202 0/08/1069392.

Goldsmith, M., \& Osman, S. (2020). Leadership in a time of crisis: The way forward in changed World. Rosetta Books.

Hall, E.T. (1976). Beyond culture. www.constantforeigner.com.

Kouzes, J.M., \& Posner, B.Z. (2017). The leadership challenge: How to make extraordinary things happen in organizations.( $6^{\text {th }}$ Ed). John Wiley \& Sons, Inc.

Littlefield, R., \& Sellnow, T. L. (2015). Risk and crisis communication: Navigating the tensions between organizations and the public. Lexington Books.

Lock, D. (2019). What is change management: The definitive guide.daniellock.com.

Mackey, S. (2015). Theorising and practicing public relations crisis management in Crisis communication in a digital world by Mark Sheenan \& Deirdre Quinn Allan (Eds.), Cambridge.

Mehta, A., \& Greer, D. A. (2015). Communicating during disasters: Insights from the 2013 Bundaberg flood in Crisis communication in a digital world by Mark Sheenan \& Deirdre Quinn Allan (Eds.), Cambridge.

Modzelewski, J. (2019). Talk is chief : Leadership, communication \& credibility in a high-stakes world.Rosetta Books.

Motschnig,, R., \& Ryback, D. (2016). Transforming communication in leadership and teamwork: person-centered innovations, Springer. 
Ndlela, M. (2019). Crisis communication: A stakeholder approach. Springer.

Northouse, P. G. (2001). Leadership theory and practice. ( $2^{\text {nd }}$ Ed.), Sage Publications Inc.

O'Neil, J. (2002). On emotional Intelligence: A conversation with daniel goleman. EBSCO Publishing.

Rathore, D., Chadha, N. K., \& Rana, S. (2017). Emotional intelligence in the workplace. Indian Journal of Positive Psychology 8(2), 162-165.

Rebrina, F.G., Khakimova, S., \& Ishkinyaeva, A. (2019).Networking cooperation in forming soft skills of a new type of teacher. Australian Educational Computing 34(1), 119.

Rogers, C. R. (1979). The foundations of the person centred approach. Education 100(2), 98107.

Sawyer, K. (2008). Group genius: The creative power of collaboration. Basic Books.

Sinha, S., \& Sinha, D. (2007). Emotional intelligence and effective communication. www.res ea rchgate.net .
Stacks, D. W., \& Wright, D. K. (Eds.) (2014). Leadership communication: How leaders communicate and how communicators lead in today's global enterprise. Business Expert Press.

Unsar, S. (2014). Leadership and Communication: A case from glass, textile, and apparel sector in Turkey. Springer.

Venus, M., Stam, D., \& Knippenberg, D.V. (2018). Research: to get people to embrace what will stay the same. Harvard Business Review.

Warwick, D. D. (2011). The urgent need for skilled transformational leaders: Integrating transformational leadership and organization development. Journal of Leadership, Accountability and Ethics 8(5), 11-26

Worley, C.G., \& Vick, Y.H. (2005). Leading and managing change. Graziadio Business Review 8(2), 1-10.

Yukl, G. (2010). Leadership in organizations. ( $\left.7^{\text {th }} \mathrm{Ed}.\right)$, Pearson.

Zaremba, A. J. (2010). Crisis communication: Theory and practice. Routledge. 